\title{
Table-top two-color soft X-ray laser from Ni-like Mo plasma
}

\author{
Davide Bleiner*a \\ ${ }^{a}$ Swiss Federal Laboratories for Materials Science \& Technology (Empa), Überlandstrasse 129, CH- \\ 8600 Dübendorf, Switzerland;
}

\begin{abstract}
Two-color laser pulses in a laboratory setup are interesting for enabling a number of advanced spectroscopy techniques. The generation by means of Ni-like laser-produced plasmas is promising for scaling down the wavelength towards the soft X-ray. The occurrence of a two-color signal has been documented in the 1990s and a detailed atomic physics study interpreted the pulse as due to concomitant collisional pumping (color-1) and self-photoexcitation (color-2). If this framework is on the atomic physics basis valid, its kinetic and hydrodynamic quantitative aspects need to be better understood. In particular, experimental observations leave room for a number of open points. Here we propose am amplified Raman scattering (ARS) scheme, to explain the growth of color-2 and its sensitivity to the plasma irradiation scheme.
\end{abstract}

Keywords: plasma, XUV, two-color, spectroscopy, collisional, photopumping, Raman

\section{INTRODUCTION}

Short-wavelength pulses with bimodal spectra ("two-color") are very insightful for spectroscopic applications, for instance to investigate the structure and dynamics of chemical processes ${ }^{1,2}$. In particular, when two coherent wavelengths with a significant spectral separation are available, a number of chemical processes can be probed, by means of pumpprobe techniques, differential absorption, stimulated scattering, and so on. Advanced spectroscopies are available at beamlines, and research is done to enable them in the home-lab. Unfortunately, advanced spectroscopies profiting from two-color pulses are still not very developed even at beamlines, due to the difficulty to generate such signals.

Just recently, SLAC researchers demonstrated pairs of X-ray laser pulses through self-amplified spontaneous emission (SASE) in a X-ray Free-Electron Laser, with wavelengths at ca. $0.81 \mathrm{~nm}$ (ca. $1530.7 \mathrm{eV}$ ) and ca. $0.83 \mathrm{~nm}$ (ca. 1493.8 $\mathrm{eV}$ ), i.e. a minimal spectral separation. Also the SACLA beamline demonstrated two-color X-ray pulses at ca. $0.09 \mathrm{~nm}$ (ca. $13^{\prime} 776 \mathrm{eV}$ ) and ca. $0.12 \mathrm{~nm}\left(10^{\prime} 332 \mathrm{eV}\right)$. Masoudnia et al. ${ }^{3}$ have summarized the three beamline architectures that are functional to generate two-color pulses, i.e. (i) double split undulator mode, (ii) gain-modulated undulator mode, and (iii) double-bunch undulator mode. Spectral bandwidth tolerances in both the source, whether the beam is seeded or not, and the detection, whether it is a high-res or a FT spectrometer, are crucial in making such effects characterizable. However promising the results, short-wavelength free-electron lasers are currently available in very few sites ${ }^{4}$. The spectroscopic potential demands that such special pulses are made available in home-lab tabletop sources, to be fully exploited 24/7.

Nilsen et al. ${ }^{5-10}$ predicted and experimentally demonstrated ${ }^{11}$ that laser-produced micro-plasmas are able to produce two-color pulses by means of amplified spontaneous emission (ASE). In the collisional excitation scheme, leading to color-1, the upper laser level cannot radiatively relax back to the ground state and is indeed metastable ${ }^{12}$. The lowest excited level in Ni-like ions ${ }^{13}$ is the $3 \mathrm{~d}^{9} 4 \mathrm{~s}^{3} \mathrm{D}_{3}$ that can decay to the ground state only by magnetic octupole (M3) transition. For Mo, its lifetime was reported to be $4^{\prime} 484 \mathrm{~ms}$. Electric-dipole forbidden transitions dominate a large region of the M-shell X-ray emission of Ni-like ions. Levels that decay by E1 transitions feature lifetimes in the ps time-scale, while the levels that decay by M1 or E2 transitions show lifetimes in the ns or $\mu$ s range. Träbert $e t a l^{12}$ also showed that $3 \mathrm{~d}^{9} 4 \mathrm{~d}$ cannot decay easily towards $3 \mathrm{~d}^{10}$ levels, and their predominant decay branch leads to the $3 \mathrm{~d}^{9} 4 \mathrm{p}$ level.

The observation of two laser colors has been widely interpreted as due to two different "population inversion" mechanisms, namely through

X-ray Lasers and Coherent X-ray Sources: Development and Applications, edited by Annie Klisnick,

Carmen S. Menoni, Proc. of SPIE Vol. 10243, 102430I · C 2017 SPIE

CCC code: $0277-786 \mathrm{X} / 17 / \$ 18 \cdot$ doi: $10.1117 / 12.2264981$ 
(i) collisional-pumping $|\mathrm{g}\rangle \rightarrow\left|\mathrm{u}_{1}\right\rangle$ (monopole selection rule $\Delta \mathrm{J}=0$ ) with color-1 lasing as $\left|\mathrm{u}_{1}>\rightarrow\right| \mathrm{l}_{1}>$;

(ii) self-photo-pumping $|\mathrm{g}>\rightarrow| \mathrm{u}_{2}>$ (dipole selection rule: $\Delta \mathrm{J}=1$ ) with "color-2 lasing" as $\left|\mathrm{u}_{2}>\rightarrow\right| \mathrm{l}_{2}>$.

In a Ni-like radiator, i.e. for $\mathrm{Z}>28$ targets, the levels refer to the following (LS-coupling) terms, with numbers referring to Fig. 5:

0. $\quad \mid \mathrm{g}>$ is the ground state $3 \mathrm{~d}^{10}(\mathrm{~J}=0)$;

1. $\quad \mid l_{1}>$ is the lower laser state $3 \mathrm{~d}^{9} 4 \mathrm{p}^{1}(\mathrm{~J}=1)$ of color-1;

$2.0 \quad \mid \mathrm{u}_{1}>$ is the upper laser state $3 \mathrm{~d}^{9} 4 \mathrm{~d}^{1}(\mathrm{~J}=0)$ of color- 1 ;

$2.1 \quad \mid l_{2}>$ is the lower laser state $3 d^{9} 4 d^{1}(\mathrm{~J}=1)$ of color-2;

$3 \mid \mathrm{u} 2>$ is the upper laser state $3 \mathrm{~d} 9 \quad 4 \mathrm{fl}(\mathrm{J}=1)$ of color-2.

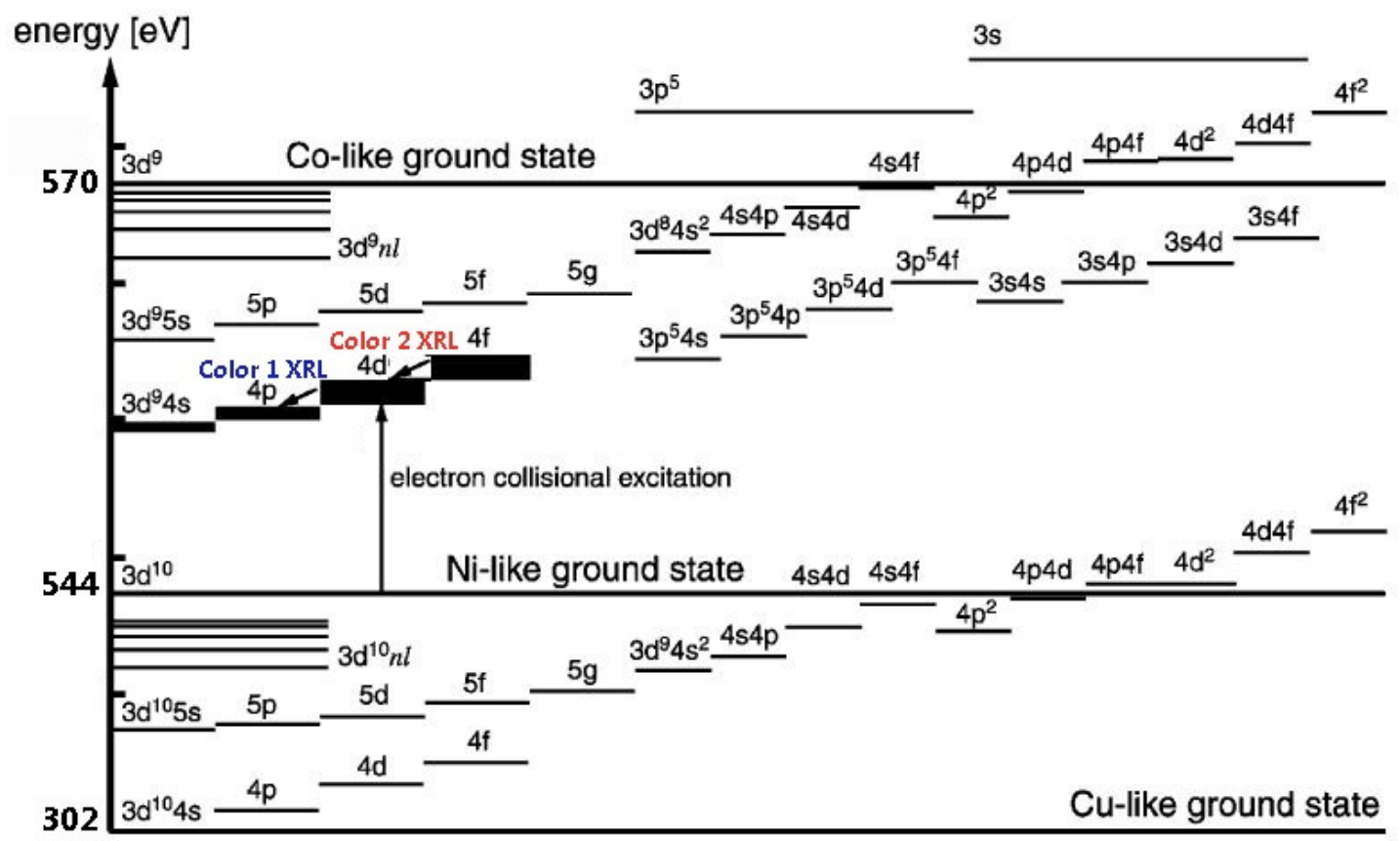

Fig. 1: Grotrian scheme of the energy levels in a Ni-like $\mathrm{Mo}^{14}$, as well as neighboring ionization stages. The color-1 collisionally pumped X-ray laser is indicated in the 4d-4p, as well as the "red-shifted" color-2 line in the 4f-4d. The Energy scale corresponds to ca. $200 \mathrm{eV}$ per tick interval. The values given in the energy axis are the ionization energies of each isoelectronic ground state

Figure 1 shows a Grotrian scheme summarizing the Ni-like levels and transitions ${ }^{15}$. It should be said that the lower laser level $\mid l_{2}>$ has substantial mixing between the LS configurations. For the case of the Ni-like Mo, the $\mid l_{2}>$ state is a mixture of $0.6614 \mathrm{~d}^{1} \mathrm{P}_{1}-0.6224 \mathrm{~d}^{3} \mathrm{P}_{1}-0.3284 \mathrm{~d}^{3} \mathrm{~S}_{1}$. For Mo this amounts to a $0.77-\mathrm{eV}$ correction ${ }^{8}$. Given the upper as well as lower population for the color-2 results from a mix of collisional and photo-driven pumping, it is possibly not accurate to speak about "self-photo-pumping" only, as found in some references ${ }^{16}$, while referring to the color-2 laser line.

The self-photopumping process requires strong re-absorption of background radiation, to induce the $|\mathrm{g}\rangle \rightarrow\left|\mathrm{u}_{2}\right\rangle$ population, inverted with respect the $\mid l_{2}>$. Re-absorption implies an optically thick plasma medium, whose optical density is significantly above 10 . Nilsen et al. ${ }^{8}$ calculated that the optical depth of the self-photopumping is 100 and the line strength is 0.0163 photons per mode, with a gain of $37 \mathrm{~cm}^{-1}$, and an electron temperature of $295 \mathrm{eV}$. 
As a matter of fact, the upper laser level $\mid \mathrm{u}_{2}>$ can be populated by collisional excitation and photoexcitation at the same time. The inversion is presumably accomplished by destroying the lower laser state $\mid l_{2}>$ by collisional mixing mainly ${ }^{5}$. Different authors agree that the thick plasma requires radiative pumping at ca. $350 \mathrm{eV}(3.53 \mathrm{~nm})^{3,7}$. Such soft X-ray pump may be calculated as the peak radiation for a black-body emitter at a temperature of $70 \mathrm{eV}$, using Wien's law. A $70 \mathrm{eV}$ plasma is not a difficult condition to attain and that is why the self-photopumped mechanism is a qualitatively plausible interpretation for sustaining the $4 \mathrm{f}$ population leading to color- 2 .

Long-range, i.e. beyond the $c \tau_{\text {pulse }}$ "horizon", optical thickness is required for the entire amplification time and space (fig. 2). Nilsen ${ }^{7}$ calculated strict spatio-temporal windows for the accomplishment of the self-photopumping, while Masoudnia et al. ${ }^{17}$ provided less strict ranges. It should be said that the laser input conditions are not exactly the same for the two references, and in this respect the two works provide comparable orders of magnitude for the temporal and spatial windows. Both agree that color-2 is characterized by a rather limited efficiency, which demands a more detailed explanation.

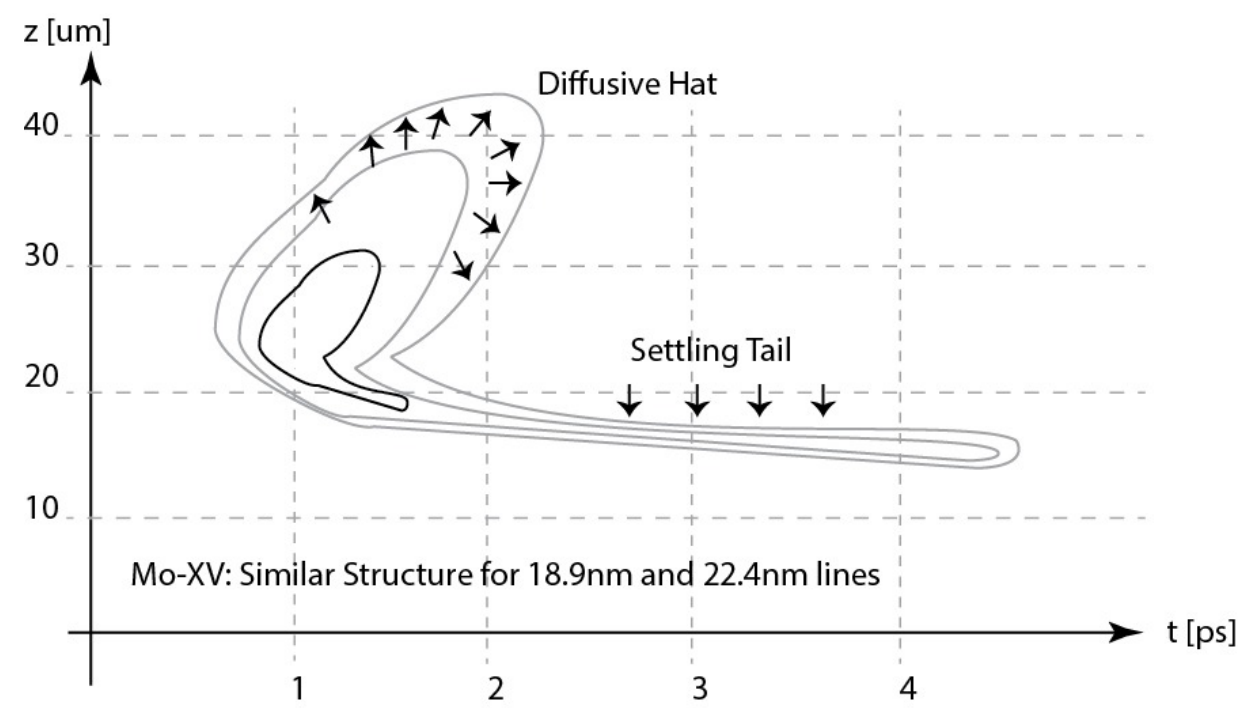

Fig. 2: Sketch of plasma structure and evolution supposed to host the collisional and the photo-pumped ASE (Plasma Zoning Model). The contour indicate density, temperature or gain. The drawing is stylized from literature results, such as Nilsen et al. $^{5-10}$ and Masoudnia ${ }^{17}$.

Two major limitations to propagating the gain through the plasma are possibly refraction and transit-time ${ }^{7}$. Nilsen however excluded that refractive effects are important as the gradients are claimed to be small ${ }^{5}$, while a few other workers do indicate that this effect must be considered carefully ${ }^{17}$. Nilsen computed the gain for a number of Ni-like Mo lines (Tab. 1, in ref. $^{7}$ ), with and without optical thickness considered in the simulation, showing that radiation selftrapping is essential for the gain of the self-photopumping $4 \mathrm{f} \rightarrow 3 \mathrm{~d}$ line ( $88 \%$ reduction when the plasma is optically thin), and that it is also important for the collisional $4 \mathrm{~d} \rightarrow 4 \mathrm{p}$ line ( $26 \%$ reduction when the plasma is optically thin). The latter suggests that (i) collisional-radiative mixing is important, and (ii) color-1 and color-2 are somehow interlinked, rather than contending pumping schemes.

Nilsen et al. ${ }^{8}$ have done a detailed gain length study of Mo plasma-laser with the traveling-wave excitation (TWE) ${ }^{18}$, and showed that without the traveling wave color-2 showed a weak gain. The use of the traveling-wave setup does increase the output of the color-2 in Mo by two orders of magnitude and is essential for observing strong lasing on this line. Furthermore, the $4 \mathrm{f} \rightarrow 4 \mathrm{~d}$ line was never observed in a non-lasing plasma, i.e. without color-1. Its interpretation is thus relying on calculations using measured values of the $3 \mathrm{~d} \rightarrow 4 \mathrm{f}$ photo-pumping resonance.

Kuba et al. ${ }^{19}$ investigated the performance of plasma-lasing under TWE and also a dual pulse scheme (70J long pulse for high pre-ionization, and 30J short pulse for inversion pumping) with fundamental $(1 \omega)$ and second harmonic $(2 \omega)$. Using TWE a 300-fold increase in intensity on color-1 and color- 2 was observed. Refraction was pointed out to be an important 
factor, with as high as $13 \mathrm{mrad}$ deflection angle over $1 \mathrm{~cm}$ plasma propagation. The lasing was observed from both endings of the plasma column. In order to prevent cold plasma termination, the line focus was longer than the target length. Lasing was observed on the color- $14 d-4 p$ line $(\lambda=13.9 \mathrm{~nm}$ with Ag target), but also on what has been computationally identified as color- $4 f-4 d(\lambda=16.0 \mathrm{~nm}$ with $\mathrm{Ag}$ target). Insightful observations are reported that turn out to be crucial, namely:

- The intensity of the color-2 was correlated to the one of the color-1, in some cases with similar amplitude, but color-2 was very unstable from shot to shot, with very high sensitivity to the energy of the short pump pulse;

- The color-2 was observed only under TWE, and in particular when TWE was progressing towards the spectrometer (forward plasma ending), but not on the opposite side;

- The temporal delay between long and short pulse must be set to $250 \mathrm{ps}$ and the plasma lengths greater than 3.8 , such that color-1 was saturated;

- The irradiation scheme must be such that $2 \omega$ for the first pulse and $1 \omega$ for the second pulse, and never with other combinations of $1 \omega$ and $2 \omega$.

Recently, Siegrist et al ${ }^{16}$ reported on two-color plasma-lasing experiments where the self-photopumped Neon-like $3 \mathrm{~d}$ ${ }^{1} \mathrm{P}_{1}-3 \mathrm{p}{ }^{1} \mathrm{P}_{1}$ and Nickel-like $4 \mathrm{f}^{1} \mathrm{P}_{1}-4 \mathrm{~d}{ }^{1} \mathrm{P}_{1}$ laser transitions were invoked. The self-photopumped $\mathrm{x}$-ray laser was regarded as an alternative scheme to electron collisional excitation in the search for an extension of available XUV and soft X-ray laser wavelengths. This implies that color-1 and color-2 are alternatives, and not interlinked. Further, one has to point out that the occurrence of the so-called "self-photo-pumped" wavelength (color-2) is "harder" (higher photon energy) than the collisional one for the Ne-like radiators, but "softer" for Ni-like case. Moreover, the color-2 gain is typically lower than color-1 although the saturation intensity is expected to be higher ${ }^{16}$. The Bern group studied the parametric dependence of self-photopumping, e.g. varying the pre-pulse delay. The latter was indicated to scale-down, for optimum operation, as a function of atomic number of the target. Two-color lasing was shown to occur for a number of "uncharted elements" including Ne-like V, Cr, Fe, and Co as well as Ni-like Ru and Pd. Time-resolved streak measurements were presented in the same work, which show that color-2 is not occurring over the entire lifetime of color-1. On the contrary, color-2 occurs, with a minimal time-lag (ca. 2ps), after and during color-1, and only in parallel to the peak of it. This observation is very insightful, although the time resolution was at the limit of the used instrumentation.

If the mechanism of the two-color process is qualitatively interpreted as due to a collisional (color-1) and a photo-excited (color-2) pumping, some quantitative aspects on the realization of the latter process remain to be clarified. In ref. ${ }^{17}$ we have discussed in detail the contribution of collisional and photo-driven pumping for color-2. Still three main "open points" deserve to be better understood, namely:

- Self-photopumping "recycles" existing energy, and it will not lead to a growth of population in the upper laser level; Assuming self-absorption supports self-photopumping, how does the color-2 stimulated emission is seeded?

- Assuming the seed of photoexcitation comes from Bremsstrahlung, the fast plasma dynamics can deplete the required radiation within the buildup time; on the other hand, if the plasma is too hot, excessive photoexcitation can progress towards photoionization;

- The observation made in Kuba et al. ${ }^{19}$ (as summarized also above) indicate a dependence on plasma structure; What is the role of TWE? What is the "interlink" between color-1 and color-2, and why the optical thickness is crucial for both of them?

Aim of this paper is to contribute to the process of two-color plasma-lasing, and discuss in further detail a potential mechanism that would quantitatively explain the occurrence of color-2, even without the strict requirement for a population inversion.

\section{MATERIALS \& METHODS}

\subsection{Experimental}

A planar molybdenum (Mo) target is line-irradiated by a $\lambda=1054 \mathrm{~nm} \mathrm{Nd:glass} \mathrm{chirped} \mathrm{pulse} \mathrm{amplification} \mathrm{(CPA)} \mathrm{laser}$ system with a pulse duration of $1.5 \mathrm{ps}$ and the plasma gain-medium is created. Two pre-pulses $(0.5 \%$ and $16 \%$ of main 
pulse energy) and a 2.5J main-pulse are delivered at an incident angle of $40^{\circ}$ with respect to the target normal (Fig. 3). The pre-pulses arrived $4.8 \mathrm{~ns}$ and $0.5 \mathrm{~ns}$ before the arrival of the main pulse. It should be remarked that is the first observation of two-color Mo-plasma laser with a pump energy below 3J. This is promising in order to enable advanced spectroscopic techniques in the home laboratory.

A flat-field grazing incidence spectrometer is used to acquire spectra of the plasma-driven XUV laser (Fig. 3). The XUV laser emission was measured on-axis using a 1'200 grooves/mm, aberration-corrected, flat-field Hitachi grating. The spectrum was recorded on a phosphor screen (P43) imaged onto a cooled CCD camera having a pixel size of $23 \mu \mathrm{m}$. The spectrometer resolves the image with a spectral resolution of $10^{-3}$ thanks to an "entrance pinhole" to delimit the aperture of the spectrometer.

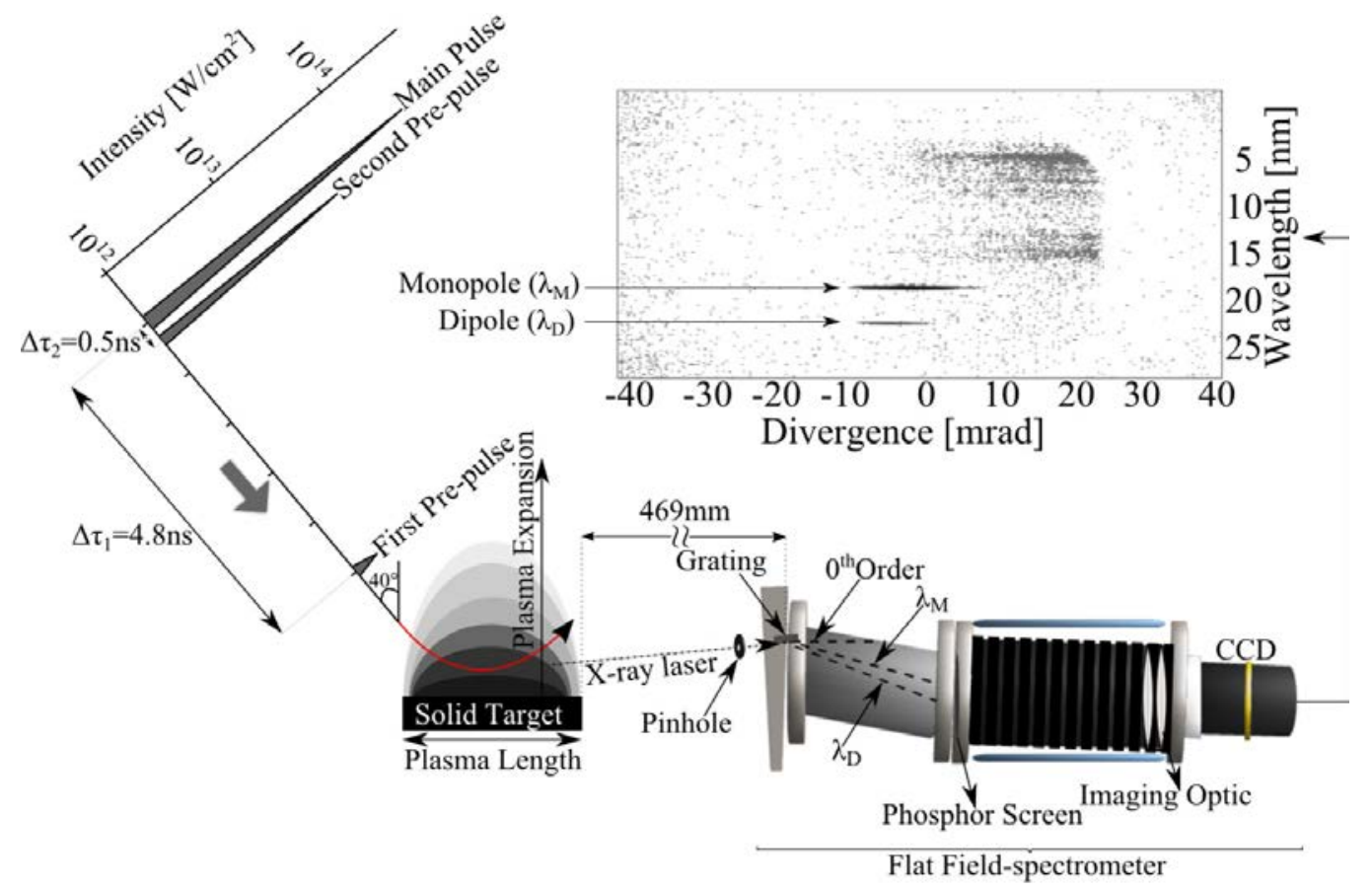

Fig. 3: Schematic of the experimental setup used for the generation and observation of the two-color Mo-plasma X-ray laser.

\subsection{Computational}

A suite of public codes was used and coupled through self-written routines to investigate amplified spontaneous emission (ASE) in Ni-like $\mathrm{Mo}^{14}$. Boundary conditions were chosen to reproduce the experimental conditions, as summarized in the section 2.1. The first code is performing 1D hydrodynamic calculations of the laser-plasma expansion. With that, one can compute the electron temperature and density, as well as the gain in the spatio-temporal dimension. Given the nature of the lasing process, of ASE along the plasma column, here a translational invariance along the propagation dimension is assumed. An atomic physics code (FAC) was used to obtain collisional and radiative rate coefficients. The exact calculation of the gain and the refractive propagation of the XUV laser was done with self-written codes. All codes are described in detail in ref. ${ }^{3}$.

\section{RESULTS \& DISCUSSION}

\subsection{Observation of two-color XUV lasing process with minimal pump energy}

Fig. 4 summarizes experimental results obtained from a Mo target. Our computational results are summarized in Tab. I, along with experimental data for the case of a Mo target. Fig. 5 shows the collisional rates as a function of electron 
temperature, along with the inversion temperature (see arrows), i.e. the temperature at which excitation and relaxation rates are equal for a given transition. Above the inversion temperature, excitation dominates, which helps to grow population at the upper level. For some transitions, one cannot find any inversion temperature because the relaxation is always larger than the excitation, and such transitions do not experience any population development.
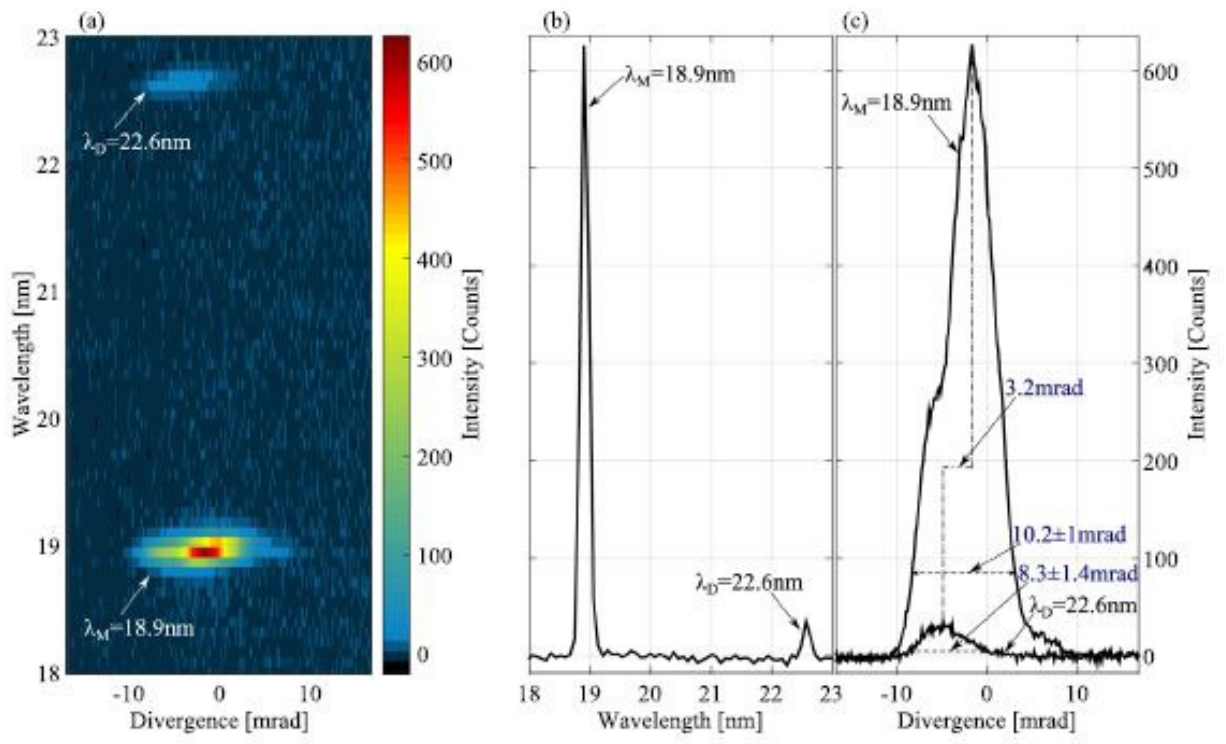

Fig. 4: Experimental data showing (a) the spectrograph image, (b) the image projection along the wavelength axis, and (c) the angle resolved image as centered on the color-1 peak.

Tab. I: Main parameter values as obtained from calculation or experiment. See text for discussion.

\begin{tabular}{|c|c|c|}
\hline Denomination (Transition) & $\begin{array}{c}4 d-4 p \\
\text { (color-1) }\end{array}$ & $\begin{array}{c}4 f-4 p \\
\text { (color-2) }\end{array}$ \\
\hline Far field beam centroid [mrad] & 0.0 & Mo: 3.2 \\
\hline Pointing stability [mrad] & Mo: 1.4 & Mo:1.4; \\
\hline Beam refractive divergence [mrad] & Calc. 6.2 (exper. Mo: 10.2) & Calc. 9.8 (exper. Mo: 8.3) \\
\hline Electron density at max gain $\left[\mathrm{cm}^{-3}\right]$ & $1.210^{20}$ & $2.110^{20}$ \\
\hline Electron temperat. at max gain $[\mathrm{eV}]$ & 295 & 350 \\
\hline Inversion electron temperature $[\mathrm{eV}]$ & 270 & 320 \\
\hline Elevation of gain peak $[\mu \mathrm{m}]$ & 76 & 70 \\
\hline Rel. time of gain peak [ps] & 0 & 0.2 \\
\hline Gain peak $\left[\mathrm{cm}^{-1}\right]$ & 230 & 55 \\
\hline Gain lifetime [ps] & 5.7 & 1.1 \\
\hline Gain thickness $[\mu \mathrm{m}]$ & 32.8 & 18.2 \\
\hline Gain buildup time [ps] & 0.7 & 0.9 \\
\hline Gain buildup length $[\mu \mathrm{m}]$ & 11.5 & 6.3 \\
\hline Intensity [cts] & Mo: 600 & Mo: 35 \\
\hline Rel. bandwidth $\Delta \lambda / \lambda 10^{-3}$ & Mo: 9 & Mo: 6 \\
\hline
\end{tabular}




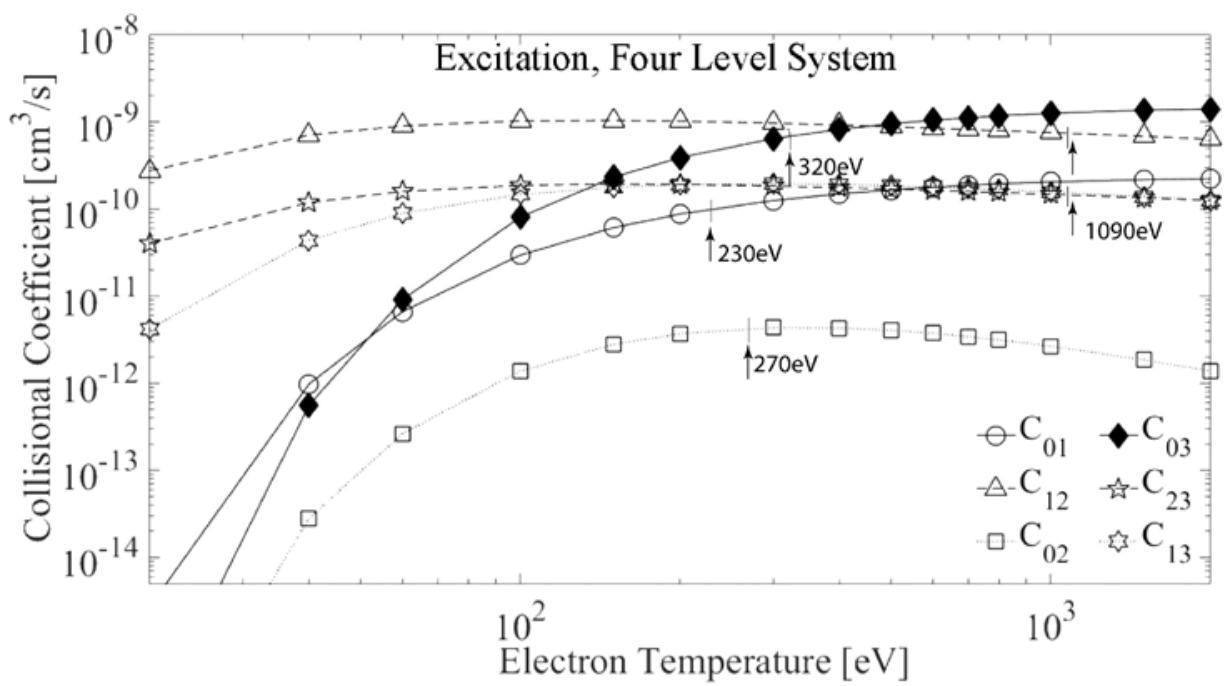

Fig. 5: Collisional rates for a selection of six transitions in the Ni-like Mo. The arrows indicate so-called "inversion temperatures". Above such temperatures the upward transitions exceed the relaxation rates, which contributes to the upper population growth. The level 2 is 2.1 (see introduction).

The inversion values indicate that the upper level are populated from the ground level $\mid \mathrm{g}>$ for typical temperatures of a few hundred $\mathrm{eV}$, while population from non-ground level require much higher temperatures above the keV. The population of the $1 l_{1}>$ level at $230 \mathrm{eV}$ (clearly for the present case of a Mo target) can degrade the lasing process, because (i) populates the low laser level thus reducing the stimulated emission rate, and (ii) subtracts electrons from the feed to the upper laser level. This temperature is thus to be intended as a bottom limit for the lasing process to be sustained.

For color-1's $\mid \mathrm{u}_{1}>$ state, the rate is low $\left(310^{-12} \mathrm{~cm}^{3} / \mathrm{s}\right)$, while the inversion dominates above $270 \mathrm{eV}$ for the considered case of a Mo target. Considering the thermal profile induced by the main pump of the laser-plasma, a low inversion temperature indicates a more rapid onset of inversion, than the "contending" color-2 process. For the latter, i.e. up to $\left|\mathrm{u}_{2}\right\rangle$, the inversion temperature is at $320 \mathrm{eV}$, i.e. $50 \mathrm{eV}$ higher than the "contending" color-1 process.

The measured Mo color-1 intensity was approximately 18 -fold stronger than color-2, despite a 2.5 order of magnitude weaker collisional rate, which suggests that the collisional gain of the color-2 is more drastically limited in duration, spatial distribution, and Stokes efficiency. The idea that the color-2 is largely sustained by radiative pumping, whose maximum rate was calculated to be $1.510^{-13} \mathrm{~s}^{-1}$, requires optical thickness (self-absorption) and rapid pumping, since, as said above, the $\mid \mathrm{u}_{2}>$ state is not metastable (actual lifetime $\tau=67 \mathrm{fs}$ ).

It is noteworthy to mention that our hydrodynamic calculation shows that in the lasing region, the population in the ground level is approximately 3.4 times higher than the population in the $\left|\mathrm{u}_{2}\right\rangle$ level. However, there is a gain because the population of the $\left|\mathrm{u}_{2}\right\rangle$ level is greater than the population of the depleted $\mid \mathrm{I}_{2}>$ level.

Fig. 6 summarizes the calculated temporal and spatial distribution of the lasing gain for the two colors from a Mo target. Temporally we determined a "gain time-voxel" (integral of gain temporal profiles) of ca. $22^{\prime} 000 \mathrm{~cm}^{-1} \mathrm{ps}$ (color-1) and ca. $250 \mathrm{~cm}^{-1}$ ps (color-2), which makes a ratio of ca. 88. Spatially we determined a "gain space-voxel" of ca. 6'600 $\mathrm{cm}^{-1}$ $\mu \mathrm{m}$ (color-1) and ca. 1'000 $\mathrm{cm}^{-1} \mu \mathrm{m}$ (color-2), which makes a ratio of ca. 6.6. This indicates that the purely collisional color-1 process benefits of a 580-fold wider "spatio-temporal window", which largely overrules the 2.5 orders of magnitude weaker collisional rate (Fig. 5). 
(a)

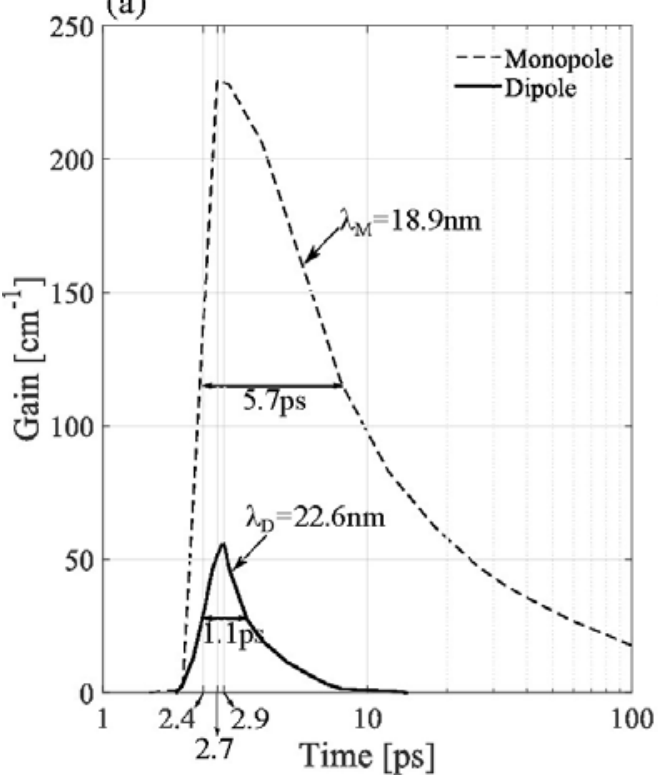

(b)

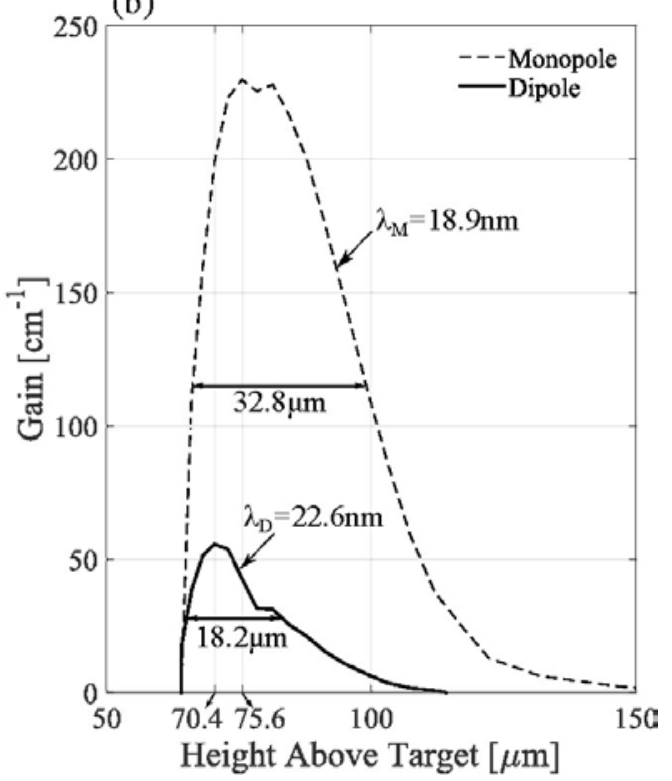

Fig. 6: Calculated ASE gain as a function of (a) time and (b) height above the target, for the color-1 (monopole) and color-2 (dipole).

In Masoudnia et al. ${ }^{17}$, we showed a substantial effect of refraction and attributed the different color-1 and color-2 divergences to the fact that color- 2 was produced deeper inside the thicker plasma, thus experiencing $20 \%$ more refraction. Scattering effects and walk-off are considered in sect. 3.2. The maximum of gain, for both colors, was calculated to occur shortly after the turning point, i.e. the deepest penetration of the pump pulse, where the density exceeds $10^{20} \mathrm{~cm}^{-3}$.

\subsection{Potential Mechanism for Color-2 Lasing, compatible with the open points}

As discussed so far, the color-2, observed in a number of specific circumstances, is atomically interpreted as due to $4 \mathrm{f}$ self-photoexcitation, while a more plasma-wise understanding is necessary. Here it is presented a complementary explanation, which fulfills all open points, but that is still subject of detailed experimental validation.

Fig. 7 was prepared with the Mo-XV (Ni-like) NIST data base, as compiled by Sugar et al. ${ }^{14}$ from a number of references. The Mo-XV (Ni-like) stage is generated with $544 \mathrm{eV}$ ionization energy, and is bounded by the $\mathrm{Cu}-$ like stage with ionization energy of $302 \mathrm{eV}$, and the Co-like limit with $570 \mathrm{eV}$, as shown in Fig. 1. Various authors compiled a consistent data base of lines, while pointing out that there is a "severe blending" of the $4 \mathrm{p}-4 \mathrm{~d}$ transition, which made only the ${ }^{3} \mathrm{G}_{5}$ level of $3 \mathrm{~d}^{9} 4 \mathrm{~d}$ clearly attributable. In Fig. 7 we manually added (gray lines) the known XUV laser $4 \mathrm{~d}$ levels, even though not reported in the NIST Database.

The strong $4 \mathrm{~d}-4 \mathrm{p}$ color- 1 emission is propagating in a high-density thick plasma-medium, where concomitantly the $3 \mathrm{~d}-4 \mathrm{f}$ transition is being self-absorbed, supporting the $4 \mathrm{f}$ population. In the optically thick plasma, the color-1 may interact with the $4 \mathrm{p}$ population and scatter. Elastic self-resonant scattering of the highly monochromatic color-1 would maintain the population inversion of the color-1 (Rabi flopping scheme), and is a trivial case. Inelastic scattering, however, over a quasi-resonant "virtual state", may create Stokes radiation that matches with color-2. The inelastic loss is highlighted in Fig. 7 at the $3 d^{9} 4 \mathrm{p}$ ensemble, which is approx. $10 \mathrm{eV}$. The Stokes branch may then be very close to the energy of color- 2 , which would support a stimulated Raman scattering process. This fast second-order process would happen in the very thick plasma-region, and would support color- 2 amplification even without the requirement of population inversion, as typical for Raman amplifiers ${ }^{20}$. The Raman process given in jj-coupling is $(5 / 2,1 / 2)_{2} \rightarrow$ virtual state $\rightarrow(1 / 2,1 / 2)_{2}$, which with $\Delta \mathrm{J}=0$ indicates a transfer of energy from the $\mathrm{j}_{1}$ core to the $\mathrm{j}_{2}$ electron. The selection rules for electronic Raman effect are discussed in Kiel et $\mathrm{al}^{22}$. Even in cases where the electric dipole transitions are strongly forbidden (weak oscillator strengths), the electronic Raman effect is quite competitive with absorption processes. It should be reminded 
that the Raman process scales with $1 / \lambda^{4}$ and thus in the XUV domain Raman is surely quantitatively significant, especially since the ASE linewidth is very narrow ${ }^{23}$.

Such process would require a strong color-1 "pump" and the concomitant photo-pumping in the same plasma domain, as in TWE, to ensure (i) hot and dense conditions over a length-scale larger than the light propagation "horizon" in the given pulse lifetime ( ps); (ii) to drive the Amplified Raman Scattering (ARS) along with color-1's ASE.

The characteristic "horizon" length-scale can be determined in TWE as $\Delta x \sim c_{p} \tau / \cos \theta$ where $c_{p}$ is the speed of light in the plasma medium, $\tau$ is the pulse duration, and $\theta$ is the grazing angle. For $1.5 \mathrm{ps}$ one can obtain a few hundred microns, which is in agreement with the prediction summarized in Fig. 2 and Fig. 6.

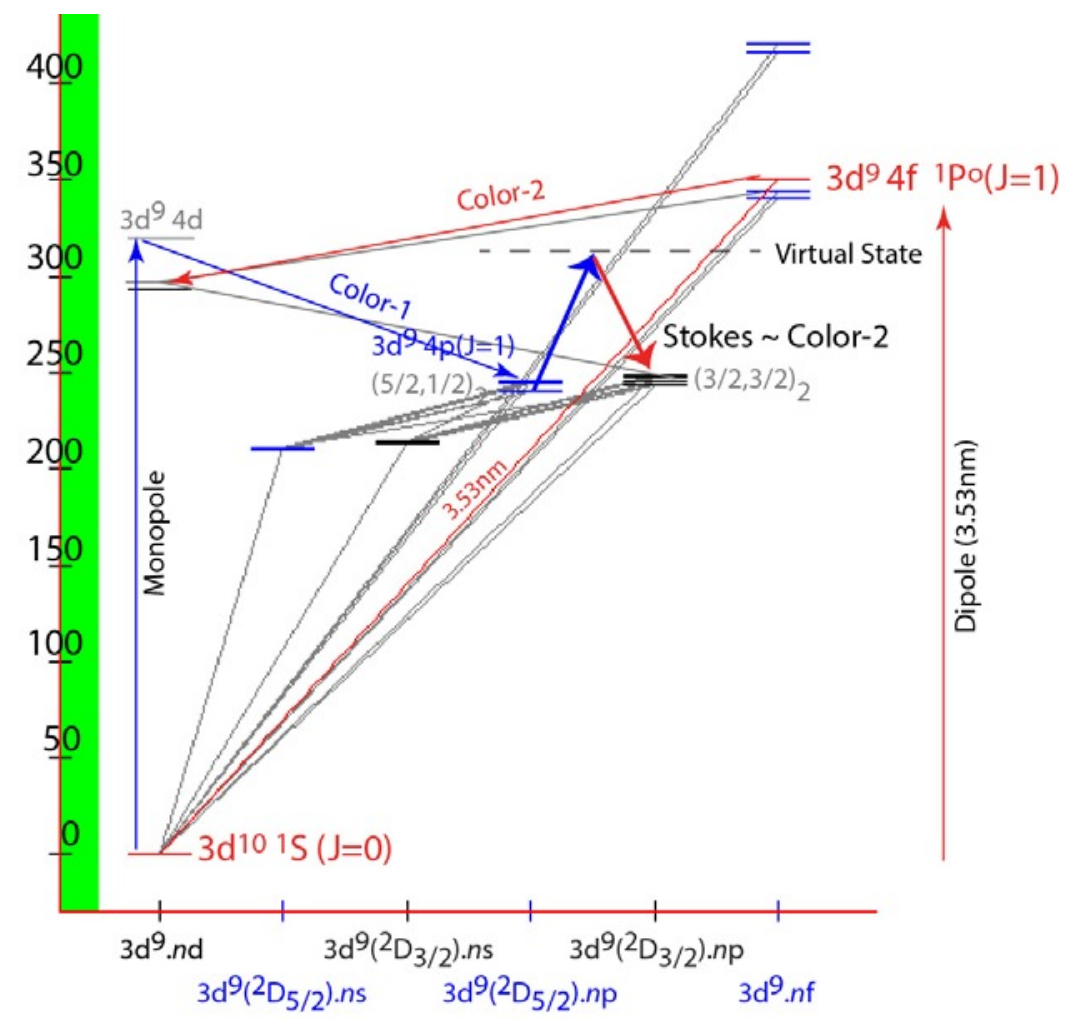

Fig. 7: Compilation of NIST Database levels for Mo-XV above the ground Ni-like level. Tentatively a Raman-like process is sketched, and with the inelastic loss close to 10eV, one can predict Amplified Raman Scattering (ARS) to support amplification of color-2, which does not require population inversion. ARS requires color-1 saturation, and links color-2 to color-1.

If a highly monochromatic ${ }^{21}$ pump field at frequency $\omega_{\text {col.1 }}$ interacts with a scattering medium (thick plasma), the spontaneous Raman scattering of photons from metastable levels, i.e. without considering coupling with color-2, can be understood as an entropic decay of highly coherent states, within energy and momentum conservation. The former implies a "conversion of color", the latter implies a walk-off of color-1 and color-2, i.e. two beam have different pointing of the centroid as observed here (as observed in this work, Tab. I). Detailed experimental investigations are however necessary, and are the subject of our ongoing work.

\section{CONCLUSIONS}

Ni-like plasmas have proven to scale down the laser wavelength of laboratory system to the extreme ultraviolet, at the limit of the soft X-rays. Further, they have demonstrated to generate two-color pulses, which are very interesting for 
spectroscopic application. The interpretation of the second color was addresses in the 1990s, considering a self-photopumping mechanism. The atomic physics of such framework is supportive of such interpretation, but kinetics, population inversion, and hydrodynamic considerations are only qualitatively addressed. In this work a complementary model is presented, which does not conflict with previous contributions on self-photoexcitation, and extends their reach in more quantitative aspects. In brief, the occurrence of Raman scattering in the thick high-density plasma, may generate a Stokes signal that is degenerate with the weak color-2. Hence, Amplified Raman Scattering (ARS) may support the growth of color-2, especially when color-1 is saturated, even without $4 \mathrm{f}-4 \mathrm{~d}$ population inversion. Color-1 and color- 2 are thus interlinked, and propagate with a walk-off, to fulfill momentum conservation. The hydrodynamic structure of the plasma as related to the pumping scheme, e.g. TWE, forward or backward TCE, etc. turns crucial to determine the efficiency of the ARS process. The initial "noise" of color-2 thus generated, could also seed the self-photopumped process in a feedback loop.

\section{REFERENCES}

[1] Marinelli, A., Ratner, D., Lutman, A. A., Turner, J., Welch, J., Decker, F.-J., Loos, H., Behrens, C., Gilevich, S., et al., "High-intensity double-pulse X-ray free-electron laser," Nat. Commun. 6, 1-6, Nature Publishing Group (2015).

[2] Marinelli, A., Lutman, A. A., Wu, J., Ding, Y., Krzywinski, J., Nuhn, H. D., Feng, Y., Coffee, R. N.., Pellegrini, C., "Multicolor operation and spectral control in a gain-modulated x-ray free-electron laser," Phys. Rev. Lett. 111(13), 1-5 (2013).

[3] Masoudnia, L., Ruiz-Lopez, M.., Bleiner, D., "Table-top two-color soft X-ray laser by means of Ni-like plasmas," Phys. Plasmas 23(4) (2016).

[4] Bleiner, D., "Comparison of laboratory-scale XUV laser with xFELs," Short-Wavelength Imaging Spectrosc. Sources 8678 (2012).

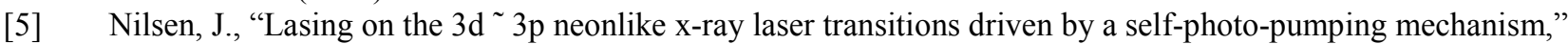
Phys. Rev. A 53(6), 4539-4546 (1996).

[6] Nilsen, J., "Resonantly Photo-Pumped Ni-like TI X-ray Laser" (1991).

[7] Nilsen, J., "Design of a picosecond-laser-driven Ni-like Mo x-ray laser near 20 nm,” J. Opt. Soc. Am. B 14(6), 1511 (1997).

[8] Nilsen, J., Dunn, J.., Osterheld, A. L., "Lasing on the self-photopumped nickel-like $4 \mathrm{f} 1 \mathrm{P} 1$ $4 \mathrm{~d} 1 \mathrm{P} 1 \mathrm{x}$-ray transition," 2677-2680 (1999).

[9] Nilsen, J., Li, Y., Lu, P.., Fill, E. E., “Self-photopumped neonlike x-ray laser,” 408-410 (1996).

[10] Nilsen, J., "Analysis of a picosecond-laser-driven Ne-like Ti x-ray laser," 3271-3274 (1997).

[11] Quan-li, D., Shou-jun, W., Zhao-hua, W.., Jing, Z., "Demonstration of Mo Soft X-Ray Lasers with a Grazing Incidence Pumping Scheme in the XL-II Facility *," 2007-2009 (2009).

[12] Träbert, E., Beiersdorfer, P., Brown, G. V., Terracol, S.., Safronova, U. I., "On the metastable level in Ni-like ions," Nucl. Instruments Methods Phys. Res. Sect. B Beam Interact. with Mater. Atoms 235(1-4), 23-27 (2005).

[13] Scofield, J. H.., MacGowan, B. J., "Energies of Nickel-like 4d to 4p Laser Lines," Phys. Scr. 46, 361 (1992).

[14] Sugar, J.., Musgrove, A., "Energy Levels of Molybdenum, Mo I through Mo XLII," J. Phys. Chem. Ref. Data 17(1), 155-239 (1988).

[15] Sasaki, A., Utsumi, T., Moribayashi, K., Kado, M., Tanaka, M., Hasegawa, N., Kawachi, T.., Daido, H., “The gain distribution of the transient collisional excited X-ray lasers,” J. Quant. Spectrosc. Radiat. Transf. 71(2-6), 665-674 (2001).

[16] Siegrist, M., Staub, F., Jia, F., Feurer, T., Balmer, J.., Nilsen, J., "Self-photopumped x-ray lasers from elements in the Ne-like and Ni-like ionization state," Opt. Commun. 382, 288-293, Elsevier (2017).

[17] Masoudnia, L., Ruiz-Lopez, M.., Bleiner, D., "Table-top two-color soft X-ray laser by means of Ni-like plasmas," Phys. Plasmas 23(4) (2016).

[18] Bleiner, D.., Feurer, T., "Pulse-front tilt for short-wavelength lasing by means of traveling-wave plasmaexcitation," Appl. Opt. 51(36), 8848-8853 (2012).

[19] Kuba, J., Klisnick, A., Ros, D., Fourcade, P., Jamelot, G., Miquel, J.-L., Blanchot, N.., Wyart, J.-F., “Two-color transient pumping in Ni-like silver at 13.9 and 16.1 nm," Phys. Rev. A 62(4), 1-7 (2000). 
[20] Premaratne, M.., Agrawal, G. P. (Govind P. ., Light propagation in gain media : optical amplifiers, Cambridge University Press (2011).

[21] Yuratich, M. A., "Effects of laser linewidth on coherent antiStokes Raman spectroscopy," Mol. Phys. 38(2), 625-655 (1979).

[22] Kiel, a.., Porto, S. P. S., "Selection rules for the electronic Raman effect," J. Mol. Spectrosc. 32(3), 458-468 (1969).

[23] Klisnick, A., Guilbaud, O., Ros, D., Cassou, K., Kazamias, S., Jamelot, G., Lagron, J. C., Joyeux, D., Phalippou, D., et al., "Experimental study of the temporal coherence and spectral profile of the $13.9 \mathrm{~nm}$ transient X-ray laser," J. Quant. Spectrosc. Radiat. Transf. 99(1-3), 370-380 (2006). 